\title{
Discovery of the Eclipsing Detached Double White Dwarf Binary NLTT 11748
}

\author{
David Kaplan $^{* a}$, J. D. R. Steinfadt ${ }^{b}$, A. Shporer ${ }^{b, c}$, L. Bildsten ${ }^{a, b}$, and S. B. Howell ${ }^{d}$ \\ ${ }^{a}$ Kavli Institute for Theoretical Physics, UC Santa Barbara, Santa Barbara, CA 93106, USA \\ ${ }^{b}$ Dept. of Physics, UC Santa Barbara, Santa Barbara, CA 93106, USA \\ ${ }^{c}$ Las Cumbres Observatory Global Telescope Network, 6740 Cortona Drive Suite 102, Santa \\ Barbara, CA 93117, USA \\ ${ }^{d}$ National Optical Astronomy Observatory, 950 North Cherry Avenue, Tucson, AZ 85719, USA \\ E-mail: dkaplan@kitp.ucsb.edu
}

\begin{abstract}
We report the discovery of the first eclipsing detached double white dwarf (WD) binary. In a pulsation search, the low-mass helium core WD NLTT 11748 was targeted for fast $(\approx 1$ minute) differential photometry with the Las Cumbres Observatory's Faulkes Telescope North. Rather than pulsations, we discovered $\approx 180$ s $3 \%-6 \%$ dips in the photometry. Subsequent radial velocity measurements of the primary white dwarf from the Keck telescope found variations with a semiamplitude $K_{1}=271 \pm 3 \mathrm{~km} \mathrm{~s}^{-1}$ and confirmed the dips as eclipses caused by an orbiting WD with a mass $M_{2}=0.648-0.771 M_{\odot}$ for $M_{1}=0.1-0.2 M_{\odot}$. We detect both the primary and secondary eclipses during the $P_{\mathrm{orb}}=5.64 \mathrm{hr}$ orbit and measure the secondary's brightness to be $3.5 \% \pm 0.3 \%$ of the primary at SDSS $-g^{\prime}$. Assuming that the secondary follows the mass-radius relation of a cold $\mathrm{C} / \mathrm{O} \mathrm{WD}$ and including the effects of microlensing in the binary, the primary eclipse yields a primary radius of $R_{1}=0.043-0.039 R_{\odot}$ for $M_{1}=0.1-0.2 M_{\odot}$, consistent with the theoretically expected values for a helium core WD with a thick, stably burning hydrogen envelope. Though nearby (at $\approx 150 \mathrm{pc}$ ), the gravitational wave strain from NLTT 11748 is likely not adequate for direct detection by the Laser Interferometer Space Antenna. Future observational efforts will determine $M_{1}$, yielding accurate WD mass-radius measurement of both components, as well as a clearer indication of the binary's fate once contact is reached.
\end{abstract}

High Time Resolution Astrophysics (HTRA) IV - The Era of Extremely Large Telescopes

May 5 - 7, 2010

Agios Nikolaos, Crete Greece

\footnotetext{
${ }^{*}$ Speaker.

${ }^{\dagger}$ Current address: Department of Physics, University of Wisconsin-Milwaukee
} 
We report the discovery of the first eclipsing detached double white dwarf (WD) binary, NLTT 11748 [1]. Originally identified as a likely low-mass WD in a binary system by Kawka $\&$ Vennes [2], our photometric observations with the 2-m Faulkes Telescope North detected both primary $(6.7 \%)$ and secondary (3.4\%) eclipses, indicating an orbital period of $5.6 \mathrm{hr}$. Subsequent spectroscopy with HIRES on Keck I found a roughly circular orbit with a radial velocity amplitude of $271 \mathrm{~km} \mathrm{~s}^{-1}$. This has since been confirmed by Kawka et al. [3] and Kilic et al. [4]. The system consists of an $\approx 0.15 M_{\odot}$ He WD photometric primary, with a $\approx 0.7 M_{\odot}$ CO WD secondary contributing $\approx 3 \%$ of the flux in $g^{\prime}$-band. Lensing of the primary by the secondary causes a $\approx 1 \%$ amplification during conjunction. We give our measured and derived parameters in Table 1.

As discussed in Steinfadt et al. [1], these observations lead to geometric constraints on the radii of both objects, including perhaps the first such constraint on a He WD. While the current constraints are not completely independent of all assumptions (having detected only a single spectral lines, we must assume a mass for the CO WD. The large radius of $\approx 0.04 R_{\odot}$ for the He WD implies that hydrogen is being burned stably, and will require a revision of the orbital evolution of such systems. NLTT 11748 will likely end up as an AM CVn system, but detailed calculations are still required.

Further constraints on the system will require additional information. With detection of the spectral lines from the secondary, the individual masses will be uniquely determined. Moreover, the gravitational redshift from the CO WD will help constrain both its mass and radius, since it can be compared to the systemic rest frame determined from the He WD. Finally, the system can be over-constrained by measuring the time delay between the primary and secondary eclipses: an effect like the Römer delay in pulsars will give rise to an $\approx 10$ s offset between the eclipse times and $1 / 2$ the orbit, as shown in Figure 1 [5].

\section{References}

[1] Steinfadt, J. D. R., Kaplan, D. L., Shporer, A., Bildsten, L., \& Howell, S. B. Discovery of the Eclipsing Detached Double White Dwarf Binary NLTT 11748, ApJ 716 (2010) L146.

[2] Kawka, A. \& Vennes, S., A new extremely low-mass white dwarf in the NLTT catalogue, A\&A $\mathbf{5 0 6}$ (2009) L25.

[3] Kawka, A., Vennes, S., \& Vaccaro, T. R. The double degenerate system NLTT 11748, A\&A 516 (2010) L7.

[4] Kilic, M., Allende Prieto, C., Brown, W. R., Agueros, M. A., Kenyon, S. J., \& Camilo, F. Accurate Masses for the Primary and Secondary in the Eclipsing White Dwarf Binary NLTT 11748, ApJ in press (2010) arXiv:1008.1069.

[5] Kaplan, D. L., Mass Constraints from Eclipse Timing in Double White Dwarf Binaries, ApJ 717 (2010) L108.

[6] Hogg, D. W., Blanton, M. R., Roweis, S. T., \& Johnston, K. V., Modeling Complete Distributions with Incomplete Observations: The Velocity Ellipsoid from Hipparcos Data, ApJ 629 (2005) 268. 


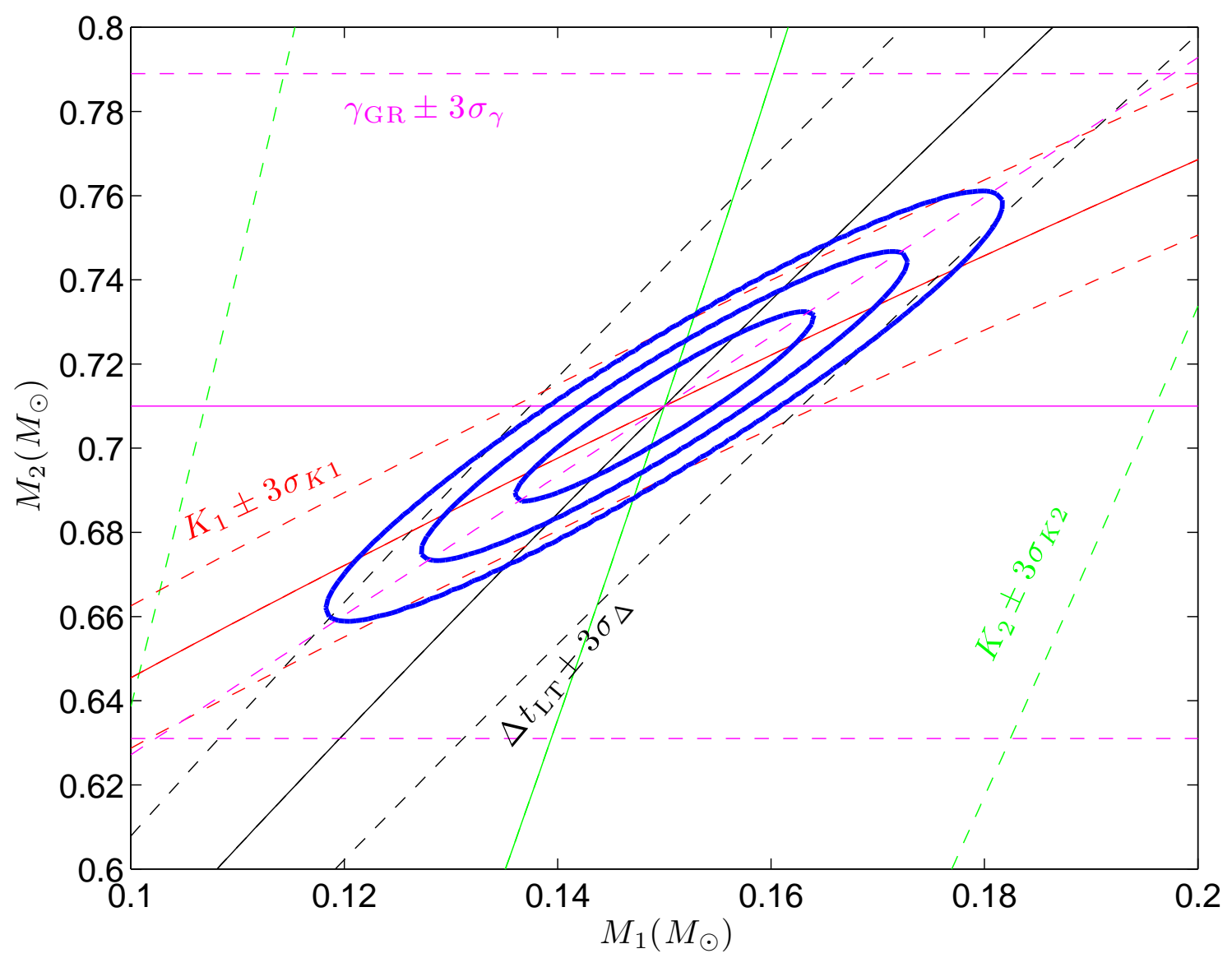

Figure 1: Constraints on individual masses from a single radial velocity constraint $K_{2}$ and a light-travel delay $\Delta t_{\mathrm{LT}}$. We show the constraints from each measurement individually (along with $3 \sigma$ ranges) as the diagonal lines: red for radial velocity, and black for time delay. The contours show $1 \sigma, 2 \sigma$, and $3 \sigma$ joint confidence contours on $M_{1}$ and $M_{2}$; their covariance is apparent. This system has parameters similar to those of NLTT 11748, and we assumed $\sigma_{K 1}=1 \mathrm{~km} \mathrm{~s}^{-1}$ and $\sigma_{\Delta}=50 \mathrm{~ms}$, which is rather optimistic. We also show possible constraints from measurement of the gravitational redshift $\gamma_{\mathrm{GR}}$ (magenta) and a second radial velocity amplitude $K_{2}$ (green), along with $\pm 3 \sigma$ intervals. Adapted from Kaplan [5]. 


\begin{tabular}{|c|c|c|c|}
\hline Quantity & \multicolumn{3}{|c|}{ Value } \\
\hline \multicolumn{4}{|l|}{ HIRES spectra: } \\
\hline Rad. Vel. Amplitude $K_{1}\left(\mathrm{~km} \mathrm{~s}^{-1}\right)$ & \multicolumn{3}{|c|}{ 271(3) } \\
\hline Systemic Radial Velocity $\left(\mathrm{km} \mathrm{s}^{-1}\right) \ldots$ & \multicolumn{3}{|c|}{$133(2)$} \\
\hline$\chi^{2} / \mathrm{DOF} \ldots \ldots \ldots \ldots \ldots \ldots \ldots$ & \multicolumn{3}{|c|}{$4.5 / 6$} \\
\hline \multicolumn{4}{|l|}{ FTN photometry: } \\
\hline Time of Primary Eclipse (BJD TDB) & \multicolumn{3}{|c|}{$2,455,196.87828(7)$} \\
\hline Period (days) .................. & \multicolumn{3}{|c|}{$0.2350606(11)$} \\
\hline Ephemeris $\chi^{2} /$ DOF $\ldots \ldots$. & \multicolumn{3}{|c|}{$3.5 / 3$} \\
\hline Primary Eclipse Depth $d_{1} \ldots \ldots$ & \multicolumn{3}{|c|}{$0.067(3)$} \\
\hline Secondary Eclipse Depth $d_{2}$ & \multicolumn{3}{|c|}{$0.034(2)$} \\
\hline Primary Eclipse Duration $\tau_{1}(\mathrm{~s}) \ldots$ & \multicolumn{3}{|c|}{$180(6)$} \\
\hline Secondary Eclipse Duration $\tau_{2}(\mathrm{~s}) \ldots$ & \multicolumn{3}{|c|}{$185(10)$} \\
\hline$F_{2} / F_{1}\left(\right.$ in SDSS-g $\left.{ }^{\prime}\right) \ldots \ldots \ldots$ & \multicolumn{3}{|c|}{$0.035(3)$} \\
\hline Out of Eclipse $\chi^{2} / \mathrm{DOF}$ & \multicolumn{3}{|c|}{$404.8 / 372$} \\
\hline \multicolumn{4}{|c|}{ Combined data (assuming Mass of Primary $M_{1}=0.15 M_{\odot}$ ): } \\
\hline Lin. Limb Darkening Coeff. $u_{\mathrm{LD}} \ldots$ & 0.0 & 0.3 & 0.5 \\
\hline Mass of Secondary $M_{2}\left(M_{\odot}\right) \ldots$ & $0.71(2)$ & $0.71(2)$ & $0.71(2)$ \\
\hline Inclination (deg) & $89.90(11)$ & $89.88(11)$ & $89.87(11)$ \\
\hline Radius of Primary $R_{1}\left(R_{\odot}\right) \ldots \ldots$ & $0.0393(9)$ & $0.0406(9)$ & $0.0415(9)$ \\
\hline$\chi^{2} / \mathrm{DOF} \ldots \ldots \ldots \ldots \ldots$ & $285.1 / 227$ & $279.5 / 227$ & $276.5 / 227$ \\
\hline Distance $(\mathrm{pc}) \ldots \ldots \ldots \ldots$ & \multicolumn{3}{|c|}{$150(32)$} \\
\hline Sys. Kin. $(U, V, W)\left(\mathrm{km} \mathrm{s}^{-1}\right) \ldots \ldots$ & \multicolumn{3}{|c|}{$(-151(9),-183(41),-34(5))$} \\
\hline
\end{tabular}

Table 1: Measured and Derived Quantities for NLTT 11748, adapted from Steinfadt et al. [1]. Quantities in parentheses are the $1-\sigma$ uncertainties on the last digit. Out of eclipse $\chi^{2}$ was determined excluding \pm 0.005 cycles around each eclipse, while eclipse $\chi^{2}$ was derived from fitting phases \pm 0.1 cycles around each eclipse. The distance was scaled from Kawka \& Vennes [2] using our $R_{1}$, and the systemic kinematics were calculated from the proper motion [2] corrected to the local standard of rest [6] and our updated distance. 